\title{
Analysis of combining ability and gene action studies for grain yield and its component traits in bread wheat utilizing line $x$ tester mating design
}

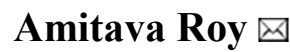 \\ Department of Genetics and Plant Breeding, College of Agriculture, Govind Ballabh Pant University of Agriculture and Technology, Pantnagar, \\ U.S. Nagar, Uttarakhand, India.

\section{Anil Kumar} \\ Department of Genetics and Plant Breeding, College of Agriculture, Govind Ballabh Pant University of Agriculture and Technology, Pantnagar, \\ U.S. Nagar, Uttarakhand, India. \\ Vaishali Rawat \\ Department of Genetics and Plant Breeding, College of Agriculture, Govind Ballabh Pant University of Agriculture and Technology, Pantnagar, \\ U.S. Nagar, Uttarakhand, India.

\section{Anu Singh} \\ Department of Genetics and Plant Breeding, College of Agriculture, Govind Ballabh Pant University of Agriculture and Technology, Pantnagar, \\ U.S. Nagar, Uttarakhand, India.
}

\begin{tabular}{|c|c|}
\hline ARTICLE INFO & ABSTRACT \\
\hline $\begin{array}{l}\text { Received : } 01 \text { July } 2021 \\
\text { Revised : } 20 \text { August } 2021 \\
\text { Accepted : } 31 \text { August } 2021 \\
\text { Available online: } 19 \text { December } 2021 \\
\text { Key Words: } \\
\text { Bread wheat } \\
\text { General combining ability } \\
\text { Grain yield } \\
\text { Line x tester cross } \\
\text { Specific combining ability }\end{array}$ & $\begin{array}{l}\text { The present research investigation was conducted in order to analyze combining } \\
\text { ability and nature of gene actions in } 33 \text { F1s of bread wheat (Triticum aestivum } \mathrm{L} \text {. } \\
\text { em. Thell) developed by crossing eleven lines with three testers in a line } x \text { tester } \\
\text { mating design in terms of grain yield and associated traits. The hybrids along } \\
\text { with the parental lines and two check varieties were planted in a randomized } \\
\text { block design in three replications. Variance ratio between general and specific } \\
\text { combining ability was found to be less than unity which indicated the prevalence } \\
\text { of non-additive gene actions involved in the inheritance of these characters. } \\
\text { Parental lines QLD } 75(3.164) \text {, followed by SOKOLL }(2.888) \text { and QLD } 65 \\
(2.819) \text { exhibited significant GCA for grain yield, while another line } \\
\text { PRL/2*PASTOR*2//FH6-1-7/3/KINGBIRD\#1//... was observed to be the better } \\
\text { general combiner for most other traits including maturity. Among the hybrids } \\
\text { cross combination NAC/TH.AC//3*PVN/3/MIRLO/BUC/4/2*PASTOR/5/... } \\
\text { HD } 3237 \text { showed significant higher positive SCA for grain yield and biological } \\
\text { yield per plant, whereas, F1 QLD } 75 \times \text { HI } 1621 \text { was a good specific combiner for } \\
\text { harvest index (\%) and number of grains per spike. The cross combination } \\
\text { VORB/4/D67.2/PARANA66.270 } \times \text { PBW } 725 \text { was observed with significant } \\
\text { higher positive SCA for days to } 75 \% \text { heading, days to maturity and plant height, } \\
\text { and another hybrid VORB/4/D67.2/PARANA } 66.270 \mathrm{H} \text { HI } 1621 \text { exhibited } \\
\text { significant positive SCA for } 1000 \text { grain weight and spike length. }\end{array}$ \\
\hline
\end{tabular}

\section{Introduction}

Wheat is one of the most extensively cultivated crops among all the food grains throughout the world ensuring the food security to nearly $35 \%$ of the global population (Bonjean et al., 2001). India holds the position of second largest producer of bread wheat globally after China having an acreage of 30.55 million hectares of land to register the alltime highest output of 107.18 million tonnes with an average national productivity hovering around

$3508 \mathrm{~kg} / \mathrm{ha}$ during rabi 2019-20 (Singh et al., 2019). With the ongoing expansion of population in the nation, there will be requirement of more than 140 million tons of wheat grain to be produced by 2050 , which is about $40 \%$ increase from our present production scenario (Singh et al., 2019). Therefore, there is an urgent requirement for development superior high yielding varieties which can exploit heterosis to break the yield ceiling. Proper

Corresponding author E-mail: amitavo66@gmail.com

Doi: https://doi.org/10.36953/ECJ.2021.22334

This work is licensed under Attribution-Non Commercial 4.0 International (CC BY-NC 4.0)

(C) ASEA 
identification of superior parents is an essential prerequisite for the development of superior high yielding varieties (Prasad, 2014). Therefore, for initiating any successful breeding programme requires the breeder to be aware of the nature of gene action and genetic system controlling the inheritance of those desired characters (Ismail, 2015). Combining ability analysis is frequently used by the breeders for evaluation of different lines in terms of their genetic value and their suitability for utilization as parents in a hybridization programme (Tabassum et al., 2017). It also assists in characterization of gene actions involved in the inheritance of various complex quantitative traits including grain yield. The knowledge of general combing ability provides the guidance to the breeder for the identification of superior performing parents which will perform better than the rest of population while utilized in a hybridization programme, whereas, measure of specific combining ability enables a breeder to select higher yielding crosses for exploitation of heterosis and non-additive portion of genetic variance.

Keeping all the previously mentioned aspects into consideration our present research experiment was aimed at investigating the general and specific combining ability variances and effects and to study the nature and magnitude of gene actions involved for yield and yield attributing characters. The analysis has been carried out as per Kempthorne (1957). Combining ability analysis in wheat has been performed earlier by Thakre et al. (1996), Kalhoro et al. (2015), Jatav et al. (2017) and Younas et al. (2020).

\section{Material and Methods}

Our present research experiment was conducted at Norman E. Borlaug Crop Research Centre, G.B. Pant University of Agriculture and Technology, Pantnagar $\left(29^{\circ} \mathrm{N}, 79.3^{\circ} \mathrm{E}\right.$ and $243.84 \mathrm{~m}$ above mean sea level) during rabi season of 2018-19 and 2019-20. Eleven genotypes of wheat were used as lines for hybridization with three male testers to produce $33 \mathrm{~F} 1 \mathrm{~s}$ during rabi season of 2018-19 (Table 1). These $33 \mathrm{~F} 1 \mathrm{~s}$ along with their parents and two standard checks viz., UP 2855 and HD 2967 were then evaluated during rabi season of 2019-20. The experiment was laid out in a randomized complete block design with three replications. Each plot within a replication consisted of two rows. Each row was one meter in length and the plants were spaced at $20 \mathrm{~cm}$ between row to row and $10 \mathrm{~cm}$ between plant to plant. Recommended cultural practices were provided accordingly to the plants. Observations were recorded on thirteen characters viz., days to $75 \%$ heading, days to maturity, number of productive tillers per plant, plant height, peduncle length, spike length, number of spikelets per spike, number of grains per spike, grain weight per spike, 1000 grain weight, biological yield per plant, grain yield per plant and harvest index (\%).

\section{Statistical Analysis}

Combining ability analysis in line $\mathrm{x}$ tester design was carried out as per the method proposed by Kempthorne (1957), which was later modified by Arunachalam (1974). Significance of combining ability is tested in terms of ' $t$ ' test as proposed by Fisher and Yates in 1938. Covariance between full sibs and covariance between half sibs were calculated from the expectations of mean squares as follows,

$$
\begin{aligned}
& \text { Cov. H. S. (line) }=\frac{M_{l}-M_{l t}}{r t} \\
& \text { Cov. H.S.(testers) } \\
& \text { Cov. H. S. }{ }_{\text {(average) }}=\frac{1}{1 t}=\frac{1}{r(2 l t-l)}\left[\frac{(l-1)\left(M_{1}\right)+(t-1)\left(M_{t}\right)}{1+t-2}-M_{l t}\right] \\
& \text { Cov. (F.S.) }= \\
& {\left[\frac{\left(M_{l}-M_{e}\right)+\left(M_{t}-M_{e}\right)}{3 r}\right]+\left[\frac{6 r \text { Cov. H.S. }-r(1+t) \text { Cov. H.S }}{3 r}\right]}
\end{aligned}
$$

Where,

$\mathrm{M}_{\mathrm{l}}=\mathrm{MS}$ line (female)

$\mathrm{M}_{\mathrm{t}}=\mathrm{MS}$ tester (male)

$\mathrm{M}_{\mathrm{lt}}=\mathrm{MS}$ due to line $\mathrm{x}$ tester

$\mathrm{M}_{\mathrm{e}}=$ error mean sum of square

Var. gca $=$ Cov. H.S $\left[\frac{(1+F)}{4}\right]$ Var. A

Therefore,

$$
\begin{aligned}
& \text { Var. A. }=\text { Cov. H. S; if } \mathrm{F}=0 \\
& \text { Var. A. }=2 \text { Cov. H. S; if } \mathrm{F}=1
\end{aligned}
$$

Var. D. $=$ Var. sca; if $\mathrm{F}=1$

Combining ability analysis is carried out utilizing 'INDOSTAT' statistical software developed at INDOSTAT Services, Hyderabad (http://indostat.software.informer.com/). ANOVA was also carried out utilizing the same software. 
Table 1: Lines, Testers and Checks utilized for the study

\begin{tabular}{|l|l|l|l|}
\hline SN & \multicolumn{1}{|c|}{ Lines } & Testers & Checks \\
\hline 1. & QBP 12-11 & $\begin{array}{l}\text { HD } \\
3237\end{array}$ & $\begin{array}{l}\text { UP } \\
2855\end{array}$ \\
\hline 2. & SOKOLL & $\begin{array}{l}\text { PBW } \\
725\end{array}$ & $\begin{array}{l}\text { HD } \\
2967\end{array}$ \\
\hline 3. & WH 1182 & HI 1621 & \\
\hline 4. & QLD 75 & & \\
\hline 5. & $\begin{array}{l}\text { PRL/2*PASTOR*2//FH6-1- } \\
7 / 3 / \text { KINGBIRD\# } 1 / / . .\end{array}$ & & \\
\hline 6. & QLD 65 & & \\
\hline 7. & NW 6036 & & \\
\hline 8. & K 1402 & & \\
\hline 9. & VORB/4/D67.2/PARANA 66.270 & & \\
\hline 10 & HPBW 01 & & \\
\hline 11. & $\begin{array}{l}\text { NAC/TH.AC//3*PVN/3/MIRLO/ } \\
\text { BUC/4/2*PASTOR/5/... }\end{array}$ & & \\
\hline
\end{tabular}

\section{Results and Discussion}

Components of variance for combining ability

Analysis of variance is carried out for all the thirteen characters at 5\% significance level. ANOVA was carried out using 'INDOSTAT' statistical software and results are tabulated in table 2. Analysis of variance revealed that mean sum of squares due to lines was significant for days to $75 \%$ maturity while mean sum of squares due to testers was found significant for number of spikes per plant. The mean sum of squares due to line $\mathrm{x}$ testers were significant for days to $75 \%$ heading, days to maturity, number of tillers per plant, spike length, biological yield per plant, number of grains per spike, 1000 grain weight, grain yield per plant, grain weight per spike and harvest index.

Analysis for genetic components of variance displayed that variance ratio between general and specific combining ability was estimated to be less than unity for all the traits under investigation except for number of spikelets per spike. This was an indicative of prevalence of nonadditive gene action involved in the inheritance of these traits, while only one trait number of spikelets per spike was governed by additive gene action Further, the dominance ratio was revealed to be less than one for the traits days to $75 \%$ heading and number of spikelets per spike indicating these traits to be governed by partial dominance, whereas, traits like number of tillers per plant, days to maturity and spike length were having dominance ratio of one and, therefore, these traits seemed to be governed by dominance. Inheritance of rest of the traits were observed to be under the control of over dominance. The similar results were observed in the earlier experimental findings of Jatav et al. (2017), Sarwar (2016) and Saeed et al. (2016).

\section{Combining ability effects}

Days to $\mathbf{7 5 \%}$ heading

GCA effects for the trait days to $75 \%$ heading varied between -2.990 to 2.677 . Out of fourteen parental lines six lines showed significant amount of GCA effects. PRL/2*PASTOR*2//FH6-1-7/3/ KINGBIRD\#1//... (-2.990) followed by K 1402 (2.101) were observed to possess highly significant negative GCA effects for the trait.

SCA effects for days to $75 \%$ heading were observed varying from -2.929 to 2.525 . Three, out of thirty three combinations possessed significant SCA effects. Hybrid VORB/4/ D67.2/PARANA66.270 x PBW 725 (-2.929) followed by HPBW 01 x HI 1621(-2.152) showed significant negative SCA effects for the trait.

\section{Days to maturity}

GCA effects for days to maturity were observed in a range from -2.455 to 2.101. Parental lines PRL/2*PASTOR*2//FH6-1-7/3/KINGBIRD\#1//... $(-2.455)$ followed by QBP 12-11 (-2.101) had high significant negative GCA effects for the trait.

For days to maturity SCA effects ranged from 4.404 to 3.717. Three cross combinations expressed significant SCA effects for the trait. The cross combination VORB/4/ D67.2/PARANA66.270 x PBW 725 (-4.404) was observed with highest significant negative SCA effects.

\section{Number of tillers per plant}

In terms of number of tillers per plant GCA effects ranged between -2.212 to 2.876 . GCA effect values were observed significant for six parents out of fourteen. Parental line QLD 65 (2.876) followed by QBP 12-11 (1.438) were observed to have high significant positive GCA effects.

SCA effects were observed from -3.368 to 3.009 for number of productive tillers per plant. Six cross combinations exhibited significant SCA effects for the trait. Hybrids HPBW 01 x HI 1621 (3.009) followed by NW $6036 \times$ HD 3237 (2.834) and QLD 75 x PBW 725 (1.979) exhibited significant positive SCA effects.

\section{Plant height (cm)}

Range of GCA effects for plant height was observed from-4.046 to 3.345. QBP 12-11 (-4.046) 
Table 2: Analysis of variance for combining ability for different characters

\begin{tabular}{|c|c|c|c|l|l|l|l|l|l|}
\hline $\begin{array}{l}\text { Source of } \\
\text { variation }\end{array}$ & $\begin{array}{l}\mathbf{d .} \\
\mathbf{f .}\end{array}$ & $\mathbf{D 7 5 H}$ & $\mathbf{D M}$ & $\mathbf{N T P P}$ & $\mathbf{P H}$ & $\mathbf{P L}$ & SL & NSPS & NGPS \\
\hline Replication & 2 & $17.707^{* *}$ & $21.586^{*}$ & $18.338^{* *}$ & $180.908^{* *}$ & $18.932^{*}$ & $1.234^{*}$ & $11.235^{* *}$ & $53.287^{* *}$ \\
\hline Crosses & 32 & $12.968^{* *}$ & $16.698^{* *}$ & $14.523^{* *}$ & 30.526 & 9.083 & $0.773^{* *}$ & $1.745^{* *}$ & $102.852^{* *}$ \\
\hline Line & 10 & $28.321^{* *}$ & 21.277 & 17.796 & 42.51 & 12.381 & 1.043 & 1.951 & 147.982 \\
\hline Tester & 2 & 6.495 & 32.131 & 35.34 & 6.545 & 8.833 & 1.357 & $6.956^{* *}$ & 60.801 \\
\hline $\begin{array}{l}\text { Line } \mathrm{x} \\
\text { Tester }\end{array}$ & 20 & $5.939^{*}$ & $12.865^{* *}$ & $10.805^{* *}$ & 26.932 & 7.459 & $0.580^{*}$ & 1.121 & $84.492^{* *}$ \\
\hline Error & 64 & 3.009 & 4.825 & 2.162 & 21.303 & 5.877 & 0.291 & 0.837 & 8.867 \\
\hline Total & 98 & 6.561 & 9.044 & 6.528 & 27.572 & 7.19 & 0.467 & 1.346 & 40.463 \\
\hline
\end{tabular}

\begin{tabular}{|l|l|l|l|l|l|l|}
\hline Source of variation & d. f. & GWPS & GW & BYPP & GYPP & HI \\
\hline Replication & 2 & 0.001 & 1.757 & $300.949^{* *}$ & $73.614^{* *}$ & 6.074 \\
\hline Crosses & 32 & $0.168^{* *}$ & $24.692^{* *}$ & $381.425^{* *}$ & $85.311^{* *}$ & $12.551^{* *}$ \\
\hline Line & 10 & 0.274 & 25.914 & 384.902 & 73.583 & 8.128 \\
\hline Tester & 2 & 0.109 & 5.953 & 527.856 & 128.513 & 12.124 \\
\hline Line $x$ Tester & 20 & $0.121^{* *}$ & $25.955^{* *}$ & $365.043^{* *}$ & $86.855^{* *}$ & $14.806^{* *}$ \\
\hline Error & 64 & 0.007 & 0.807 & 48.888 & 7.814 & 2.375 \\
\hline Total & 98 & 0.059 & 8.626 & 162.616 & 34.462 & 5.773 \\
\hline
\end{tabular}

was observed with significant high negative GCA effects for the trait. Parental line NAC/TH.AC//3*PVN/3/MIRLO/BUC/4/2*PASTO $\mathrm{R} / 5 / \ldots$ (3.226) possessed significant higher positive GCA for the trait.

SCA effects for plant height varied from -4.255 to 6.694. Significant positive SCA effects were observed in the cross VORB/4/D67.2/PARANA66.270 x HI 1621 (6.694). None of the cross exhibited significant negative SCA effects for the trait.

\section{Peduncle length $(\mathrm{cm})$}

In terms of peduncle length the GCA effect values ranged from -1.625 to 1.810 . Significant negative GCA effects were observed in the line QLD 75 (1.625), whereas, the parental line PRL/2*PASTOR*2/FH6-1-7/3/KINGBIRD\#1//...

(1.810) was observed with highest significant positive GCA for the trait.

SCA effects for peduncle length varied from 2.089 to 3.260 among the crosses. Out of thirty three F1s, only one cross combination VORB/4/D67.2/PARANA66.270 x HI 1621 (3.260) exhibited significant positive SCA effect.

Spike length (cm)

GCA effects for spike length varied from -0.457 to 0.557 .

Parental

lines

NAC/TH.AC//3*PVN/3/MIRLO/BUC/4/2*PASTO

$\mathrm{R} / 5 / \ldots(0.557)$ and PRL/2*PASTOR*2// FH6-17/3/KINGBIRD\#1//... (0.482) exhibited significant positive GCA effects.

SCA effects among the crosses ranged from -0.889 to 0.618 . None of the hybrids exhibited significant positive SCA effects for the trait.

Number of spikelets per spike

GCA effects for the trait number of spikelets per spike ranged from -0.895 to 0.366 . None of the lines had significant positive GCA for the trait. Among the testers HD 3237 (0.366) exhibited positive GCA effects.

SCA effects for number of spikelets per spike ranged from -1.068 to 1.229 . Hybrid HPBW $01 \mathrm{x}$ PBW 725 (1.229) exhibited significant positive SCA effects for the trait.

\section{Number of grains per spike}

The values of GCA effects in terms of number of grains per spike varied from -4.777 to 7.396 . HPBW 01 (7.396), followed by NW 6036 (5.919) showed high significant positive GCA effects for the trait.

SCA effects for number grains per spike were varying from -7.003 to 8.289 . Among thirty three hybrids seventeen showed significant SCA effects for the trait. The cross combinations QLD $75 \times \mathrm{HI}$ 1621(8.289) followed by NW 6036 X HI 1621 (7.034) exhibited high significant positive estimates of SCA effects.

\section{Grain weight per spike (g)}

GCA effects for the trait grain weight per spike ranged from -0.165 to 0.325 . Among the parental 
Table 3. GCA effects of the parents for different characters

\begin{tabular}{|c|c|c|c|c|c|c|c|}
\hline Parents & D75H & DM & NTPP & PH & $\mathbf{P L}$ & SL & NSPS \\
\hline 1.QBP 12-11 & $-1.990 * *$ & $-2.121 * *$ & $1.438^{* *}$ & $-4.046 * *$ & -1.067 & -0.261 & $-0.895^{* *}$ \\
\hline 2.SOKOLL & 0.566 & 0.545 & -0.029 & -0.054 & -0.517 & 0.263 & -0.463 \\
\hline 3.WH 1182 & $2.677 * *$ & $1.879^{*}$ & 0.476 & 0.056 & -1.419 & -0.052 & 0.464 \\
\hline 4.QLD 75 & $2.010 * *$ & 1.545 & 0.788 & -2.586 & $-1.625^{*}$ & -0.24 & 0.328 \\
\hline 5.PRL/2*PASTOR $* 2 /$ FH6-1-7/3/KINGBIRD\#1// .. & $-2.990 * *$ & $-2.455 * *$ & -0.657 & -0.48 & $1.810^{*}$ & $0.482 * *$ & 0.07 \\
\hline 6. QLD 65 & 0.677 & -0.232 & $2.876^{* *}$ & 0.26 & -0.395 & $-0.424 *$ & 0.456 \\
\hline 7.NW 6036 & $1.455^{*}$ & $2.101 * *$ & -0.668 & 0.585 & -0.324 & $-0.457 *$ & 0.371 \\
\hline 8. K 1402 & $-2.101 * *$ & -1.343 & 0.135 & -1.122 & 1.407 & -0.063 & -0.294 \\
\hline 9.VORB/4/D67.2/PARANA 66.270 & -0.101 & -0.566 & $-2.212 * *$ & $3.345^{*}$ & 1.374 & 0.159 & 0.255 \\
\hline 10. HPBW 01 & -0.212 & 0.101 & -0.579 & 0.817 & 0.429 & 0.037 & 0.231 \\
\hline 11.NAC/TH.AC//3*PVN/3/MIRLO/BUC/4/2*PASTOR/5/... & 0.01 & 0.545 & $-1.568 * *$ & $3.226^{*}$ & 0.327 & $0.557 * *$ & -0.524 \\
\hline SE of gi & 0.5732 & 0.7855 & 0.5414 & 1.4166 & 0.7678 & 0.1786 & 0.3057 \\
\hline SE of gi- gj & 0.8106 & 1.1109 & 0.7656 & 2.0033 & 1.0859 & 0.2525 & 0.4323 \\
\hline 12.HD 3237 & 0.475 & $0.949^{*}$ & 0 & 0.257 & 0.54 & 0.156 & $0.366^{*}$ \\
\hline 13.PBW 725 & -0.404 & 0.071 & $-1.035^{* *}$ & -0.514 & -0.048 & $-0.229 *$ & $-0.515 * *$ \\
\hline 14.HI 1621 & -0.071 & $-1.020^{*}$ & $1.035^{* *}$ & 0.257 & -0.492 & 0.073 & 0.149 \\
\hline SE of gi & 0.2993 & 0.4102 & 0.2827 & 0.7398 & 0.401 & 0.0933 & 0.1596 \\
\hline SE of gi $-g j$ & 0.4233 & 0.5801 & 0.3998 & 1.0462 & 0.5671 & 0.1319 & 0.2258 \\
\hline
\end{tabular}

Table 3. contd...

\begin{tabular}{|c|c|c|c|c|c|c|}
\hline Parents & NGPS & GWPS & GW & BYPP & GYPP & HI \\
\hline 1. QBP $12-11$ & $-4.777 * *$ & $-0.165^{* *}$ & -0.459 & $6.075^{*}$ & $2.730^{*}$ & 0.694 \\
\hline 2. SOKOLL & -0.627 & $-0.142 * *$ & $1.655^{* *}$ & 3.675 & $2.888^{* *}$ & $1.392^{* *}$ \\
\hline 3. WH 1182 & -0.648 & 0.048 & $-2.029 * *$ & -0.925 & 0.817 & 0.484 \\
\hline 4. QLD 75 & $-3.121 * *$ & $0.325 * *$ & $1.839 * *$ & $7.325^{*}$ & $3.164 * *$ & $1.352 * *$ \\
\hline 5. $\mathrm{PRL} / 2 *$ PASTOR $* 2 / \mathrm{FH} 6-1-7 / 3 / \mathrm{KINGBIRD} \# 1 / / \ldots$ & -0.522 & $-0.142 * *$ & $-1.657 * *$ & -0.708 & -0.553 & -0.354 \\
\hline 6. QLD 65 & -0.952 & $-0.210 * *$ & $1.593 * *$ & $11.164 * *$ & $2.819^{* *}$ & -0.652 \\
\hline 7. NW 6036 & $5.919 * *$ & $-0.102 * *$ & -0.436 & -2.64 & -0.288 & 0.613 \\
\hline 8. K 1402 & $-2.018^{*}$ & $0.151 * *$ & $-1.862 * *$ & -0.622 & -0.216 & -0.355 \\
\hline 9. VORB/4/D67.2/PARANA 66.270 & $-4.558 * *$ & $0.075^{* *}$ & $-0.914 *$ & $-10.177 * *$ & $-5.389 * *$ & $-1.350^{* *}$ \\
\hline 10. HPBW 01 & $7.396^{* *}$ & -0.048 & -0.596 & $-6.586 *$ & $-3.104 * *$ & $-0.990^{*}$ \\
\hline 11. NAC/TH.AC//3*PVN/3/MIRLO/BUC/4/2*PASTOR/5/... & $3.909^{* *}$ & $0.210^{* *}$ & $2.865^{* *}$ & $-6.581 *$ & $-2.868 * *$ & -0.834 \\
\hline SE of gi & 0.908 & 0.0273 & 0.3561 & 2.7619 & 1.036 & 0.4669 \\
\hline SE of gi- gj & 1.2841 & 0.0386 & 0.5036 & 3.906 & 1.4652 & 0.6603 \\
\hline 12. HD 3237 & $1.555^{* *}$ & $-0.063 * *$ & -0.141 & 1.796 & -0.053 & -0.342 \\
\hline 13. PBW 725 & -0.947 & $0.049 * *$ & $0.477 *$ & $-4.583 * *$ & $-1.947 * *$ & -0.358 \\
\hline 14. HI 1621 & -0.608 & 0.014 & -0.336 & 2.786 & $1.999 * *$ & $0.700^{* *}$ \\
\hline SE of gi & 0.4742 & 0.0143 & 0.186 & 1.4424 & 0.5411 & 0.2438 \\
\hline SE of gi $-\mathrm{gj}$ & 0.6706 & 0.0202 & 0.263 & 2.0398 & 0.7652 & 0.3448 \\
\hline
\end{tabular}

$*, * *$ significant at $5 \%$ and $1 \%$ probability levels, respectively. D75H $=$ Days to $75 \%$ heading, DM = Days to maturity, NTPP $=$ Number of tillers per plant, $\mathrm{PH}=\mathrm{Plant}$ height, $\mathrm{PL}=\mathrm{Peduncle}$
length, SL $=$ Spike length, NSPS $=$ Number of spikelets per spike, NGPS= Number of grains per spike, GWPS $=$ Grain weight per spike, GW= 1000 grain weight, BYPP = Biological yield per plant, GYPP = Grain yield per plant, $\mathrm{HI}=$ Harvest index. 
Table 4: General and specific combining ability variances

\begin{tabular}{|c|c|c|c|c|c|}
\hline SN & Characters & $\sigma_{\mathrm{GCA}}^{2}$ & $\sigma_{\text {SCA }}^{2}$ & $\sigma_{\mathrm{GCA}}^{2} / \sigma_{\mathrm{SCA}}^{2}$ & $\begin{array}{l}\text { Degree of dominance } \\
\qquad(\mathrm{H} / \mathrm{D})^{1 / 2}\end{array}$ \\
\hline 1 & Days to $75 \%$ heading & $0.6881^{* *}$ & $0.9941^{*}$ & 0.692 & 0.8499 \\
\hline 2 & Days to maturity & $1.0072 * *$ & $2.4372 * *$ & 0.413 & 1.0999 \\
\hline 3 & Number of tillers per plant & $1.1395 * *$ & $2.7224 * *$ & 0.418 & 1.0929 \\
\hline 4 & Plant height $(\mathrm{cm})$ & 0.308 & 2.9574 & 0.104 & 2.1912 \\
\hline 5 & Peduncle length $(\mathrm{cm})$ & 0.252 & 0.718 & 0.351 & 1.192 \\
\hline 6 & Spike length $(\mathrm{cm})$ & 0.043 & 0.098 & 0.439 & 1.06 \\
\hline 7 & Number of spikelets per spike & 0.172 & 0.093 & 1.849 & 0.521 \\
\hline 8 & Number of grains per spike & 4.618 & 25.691 & 0.180 & 1.668 \\
\hline 9 & Grain weight per spike (g) & 0.009 & 0.038 & 0.237 & 1.47 \\
\hline 10 & 1000 grain weight $(\mathrm{g})$ & 0.704 & 8.271 & 0.085 & 2.423 \\
\hline 11 & Biological yield per plant $(\mathrm{g})$ & 18.463 & 98.796 & 0.187 & 1.636 \\
\hline 12 & Grain yield per plant $(\mathrm{g})$ & 4.352 & 25.732 & 0.169 & 1.719 \\
\hline 13 & Harvest Index (\%) & 0.389 & 4.281 & 0.091 & 2.347 \\
\hline
\end{tabular}

Table 5: Parental lines and crosses with highest GCA and SCA effects for different characters

\begin{tabular}{|c|c|c|c|}
\hline SN & Characters & Best general combiner & Best specific combiner \\
\hline 1 & Days to $75 \%$ heading & PRL/2*PASTOR*2//FH6-1-7/3/KINGBIRD\#1//...(-2.990) & VORB/4/D67.2/PARANA66.270 x PBW 725 (-2.929) \\
\hline 2 & Days to maturity & PRL/2*PASTOR*2//FH6-1-7/3/KINGBIRD\#1//... (-2.455) & VORB/4/D67.2/PARANA66.270 x PBW 725 (-4.404) \\
\hline 3 & Number of tillers per plant & QLD $65(2.876)$ & HPBW 01 x HI 1621 (3.009) \\
\hline 4 & Plant height $(\mathrm{cm})$ & QBP 12-11 (-4.046) & VORB/4/D67.2/PARANA 66.270 x PBW 725 (-4.255) \\
\hline 5 & Peduncle length $(\mathrm{cm})$ & PRL/2*PASTOR $* 2 / /$ FH6-1-7/3/KINGBIRD\#1//... (1.810) & VORB/4/D67.2/PARANA 66.270 x HI 1621 (3.26) \\
\hline 6 & Spike length $(\mathrm{cm})$ & NAC/TH.AC//3*PVN/3/MIRLO/BUC/4/2*PASTOR/5/...(0.557) & QBP 12-11 X HD $3237(0.614)$ \\
\hline 7 & Number of spikelets per spike & HD $3237(0.366)$ & HPBW $01 \times$ PBW $725(1.229)$ \\
\hline 8 & Number of grains per spike & HPBW $01(7.396)$ & QLD 75 X HI1621 (8.289) \\
\hline 9 & Grain weight per spike $(\mathrm{g})$ & QLD $75(0.325)$ & K 1402 x HD $3237(0.392)$ \\
\hline 10 & 1000 grain weight $(\mathrm{g})$ & NAC/TH.AC//3*PVN/3/MIRLO/BUC/4/2*PASTOR/5/...(2.865) & VORB/4/D67.2/PARANA 66.270 x HI 1621 (5.464) \\
\hline 11 & Biological yield per plant ( $g$ ) & QLD 65 (11.164) & $\begin{array}{l}\text { NAC/TH.AC//3*PVN/3/MIRLO/BUC/4/2*PASTOR/5/... } \\
\text { x HD } 3237(18.054)\end{array}$ \\
\hline 12 & Grain yield per plant (g) & QLD 75 (3.164) & $\begin{array}{l}\text { NAC/TH.AC//3*PVN/3/MIRLO/BUC/4/2*PASTOR/5/...x } \\
\text { HD } 3237(9.237)\end{array}$ \\
\hline 13 & Harvest Index $(\%)$ & SOKOLL (1.392) & QLD $75 \times$ HI1621 (2.806) \\
\hline
\end{tabular}


Table 6: SCA effects of crosses for different characters

\begin{tabular}{|c|c|c|c|c|c|c|c|}
\hline Crosses & D75H & DM & NTPP & PH & PL & SL & NSPS \\
\hline QBP 12-11 X HD3237 & 0.414 & -2.061 & -1.472 & -2.231 & 0.009 & 0.614 & -0.295 \\
\hline QBP 12-11 x PBW725 & -0.374 & $3.152 *$ & 0.662 & 1.4 & -0.56 & 0.276 & 0.58 \\
\hline QBP 12-11 X HI1621 & -0.04 & -1.091 & 0.809 & 0.832 & 0.551 & $-0.889 * *$ & -0.285 \\
\hline SOKOLL X HD3237 & -0.141 & -1.061 & 1.062 & 0.282 & 0.37 & -0.15 & 0.755 \\
\hline SOKOLL X PBW725 & -0.596 & -0.848 & -1.538 & -2.603 & -0.322 & -0.324 & -0.509 \\
\hline SOKOLL X HI1621 & 0.737 & 1.909 & 0.476 & 2.321 & -0.048 & 0.474 & -0.245 \\
\hline WH1182 X HD3237 & -0.586 & -1.394 & $-2.144^{*}$ & 0.203 & -0.962 & -0.179 & -0.287 \\
\hline WH1182 XPBW725 & 0.96 & 0.818 & 0.89 & 3.171 & 0.259 & -0.293 & -0.126 \\
\hline WH1182 X HI1621 & -0.374 & 0.576 & 1.254 & -3.374 & 0.703 & 0.472 & 0.413 \\
\hline QLD75 X HD3237 & 0.747 & 0.273 & -0.555 & -3.4 & -0.322 & -0.057 & -0.076 \\
\hline QLD75 X PBW725 & -1.04 & -0.848 & $1.979 *$ & 0.006 & -1.785 & -0.088 & -0.31 \\
\hline QLD75 X HI1621 & 0.293 & 0.576 & -1.424 & 3.393 & 2.106 & 0.145 & 0.386 \\
\hline PRL/2*PASTOR*2//FH6-1-7/3/KINGBIRD\#1//... X HD3237 & -0.253 & 1.939 & -0.444 & 0.752 & -0.816 & 0.188 & 0.078 \\
\hline PRL/2*PASTOR*2//FH6-1-7/3/KINGBIRD\#1//... X PBW725 & -0.04 & -1.515 & -0.91 & -0.431 & 1.125 & 0.007 & -0.076 \\
\hline PRL/2*PASTOR $* 2 / / F H 6-1-7 / 3 / K I N G B I R D \# 1 / / \ldots$ X HI1621 & 0.293 & -0.424 & 1.354 & -0.321 & -0.309 & -0.195 & -0.002 \\
\hline QLD65 X HD3237 & 0.414 & 1.051 & -0.144 & 1.102 & 1.225 & -0.006 & 0.73 \\
\hline QLD65 X PBW725 & 0.293 & 0.263 & -1.51 & 1.684 & 0.962 & -0.171 & -0.522 \\
\hline QLD65 X HI1621 & -0.707 & -1.313 & 1.654 & -2.786 & -2.187 & 0.177 & -0.208 \\
\hline NW6036 X HD3237 & -1.697 & 0.051 & $2.834 * *$ & 1.804 & -0.007 & 0.077 & -0.728 \\
\hline NW6036 X PBW725 & 0.515 & 0.929 & 0.535 & 1.317 & 1.78 & 0.046 & 0.103 \\
\hline NW6036 X HI1621 & 1.182 & -0.98 & $-3.368^{* *}$ & -3.121 & -1.774 & -0.123 & 0.625 \\
\hline K1402 X HD3237 & -1.808 & -0.505 & 0.874 & 1.4 & 1.273 & -0.15 & -0.286 \\
\hline K1402 X PBW725 & 1.737 & 1.04 & 0.465 & 0.826 & 0.817 & -0.131 & -0.387 \\
\hline K1402 X HI1621 & 0.071 & -0.535 & -1.338 & -2.226 & -2.089 & 0.282 & 0.673 \\
\hline VORB/4/D67.2/PARANA 66.270 X HD3237 & $2.525^{*}$ & $3.717^{* *}$ & -0.055 & -2.439 & -0.968 & 0.027 & 0.228 \\
\hline VORB/4/D67.2/PARANA 66.270 X PBW725 & $-2.929 * *$ & $-4.404 * *$ & 0.812 & -4.255 & -2.292 & -0.121 & -0.313 \\
\hline VORB/4/D67.2/PARANA 66.270 X HI1621 & 0.404 & 0.687 & -0.757 & $6.694 * *$ & $3.260^{*}$ & 0.094 & 0.085 \\
\hline HPBW01 X HD3237 & 1.303 & 0.051 & -1.855 & 0.572 & 0.108 & $-0.684^{*}$ & -0.161 \\
\hline HPBW01 X PBW725 & 0.848 & 1.596 & -1.154 & 0.91 & -0.38 & 0.351 & $1.229 *$ \\
\hline HPBW01 X HI1621 & $-2.152 *$ & -1.646 & $3.009 * *$ & -1.482 & 0.272 & 0.333 & $-1.068^{*}$ \\
\hline NAC/TH.AC//3*PVN/3/MIRLO/BUC/4/2*PASTOR/5/... X HD 3237 & -0.919 & -2.061 & $1.900^{*}$ & 1.954 & 0.089 & 0.321 & 0.042 \\
\hline NAC/TH.AC//3*PVN/3/MIRLO/BUC/4/2*PASTOR/5/... X PBW725 & 0.626 & -0.182 & -0.232 & -2.025 & 0.397 & 0.449 & 0.331 \\
\hline NAC/TH.AC//3*PVN/3/MIRLO/BUC/4/2*PASTOR/5/... X HI1621 & 0.293 & 2.242 & -1.668 & 0.071 & -0.486 & $-0.770 *$ & -0.373 \\
\hline CD95\%SCA & 1.983 & 2.718 & 1.873 & 4.902 & 2.657 & 0.618 & 1.058 \\
\hline SE of SCA & 0.9928 & 1.3605 & 0.9377 & 2.4536 & 0.3093 & 0.5294 & 4.7838 \\
\hline $\mathrm{SE}\left(\mathbf{s}_{\mathrm{IJ}}-\mathbf{s}_{\mathrm{kl}}\right)$ & 1.404 & 1.9241 & 1.3261 & 3.4699 & 0.4374 & 0.7488 & 6.7654 \\
\hline $\mathrm{SE}\left(\mathbf{s}_{\mathrm{IJ}}-\mathbf{s}_{\mathrm{ik}}\right)$ & 2.8081 & 3.8482 & 2.6522 & 6.9398 & 0.8748 & 1.4975 & 13.5307 \\
\hline
\end{tabular}


Table 6 contd...

\begin{tabular}{|c|c|c|c|c|c|c|}
\hline Crosses & NGPS & GWPS & GW & GYPP & BYPP & HI \\
\hline QBP 12-11 X HD3237 & $-4.483 * *$ & -0.083 & $2.689 * *$ & -0.8 & -8.969 & $2.096^{*}$ \\
\hline QBP 12-11 x PBW725 & $3.976^{*}$ & $0.192 * *$ & $-2.270 * *$ & -2.22 & 1.994 & $-2.596 * *$ \\
\hline QBP 12-11 X HI1621 & 0.507 & $-0.109^{*}$ & -0.419 & 3.02 & 6.975 & 0.5 \\
\hline SOKOLL X HD3237 & 1.401 & $0.214 * *$ & $3.071 * *$ & 2.331 & 4.831 & 0.314 \\
\hline SOKOLL X PBW725 & $5.603 * *$ & $-0.158 * *$ & $-2.181 * *$ & $-7.095 * *$ & $-9.740 *$ & $-2.907 * *$ \\
\hline SOKOLL X HI1621 & $-7.003 * *$ & -0.057 & -0.891 & $4.763 *$ & 4.908 & $2.592 * *$ \\
\hline WH1182 X HD3237 & 2.746 & $-0.162 * *$ & -0.021 & $-7.250 * *$ & $-10.152 *$ & $-3.358 * *$ \\
\hline WH1182 XPBW725 & -1.203 & 0.076 & $2.057 * *$ & $4.763 *$ & 8.177 & $2.251 * *$ \\
\hline WH1182 X HI1621 & -1.543 & 0.085 & $-2.036^{* *}$ & 2.487 & 1.975 & 1.107 \\
\hline QLD75 X HD3237 & -2.719 & $-0.202 * *$ & $-1.464 *$ & $-4.907 * *$ & -1.785 & $-2.412 * *$ \\
\hline QLD75 X PBW 725 & $-5.570 * *$ & 0.001 & 1.001 & $3.871 *$ & $10.010^{*}$ & -0.394 \\
\hline QLD75 X HI1621 & $8.289 * *$ & $0.201 * *$ & 0.463 & 1.037 & -8.225 & $2.806 * *$ \\
\hline PRL/2*PASTOR*2//FH6-1-7/3/KINGBIRD\#1//... X HD3237 & -1.03 & 0.028 & $-3.223 * *$ & -2.943 & -9.335 & -0.053 \\
\hline PRL/2*PASTOR*2//FH6-1-7/3/KINGBIRD\#1//... X PBW725 & -1.188 & $-0.181 * *$ & $2.865^{* *}$ & -1.324 & -6.323 & 0.839 \\
\hline PRL/2*PASTOR*2//FH6-1-7/3/KINGBIRD\#1//... X HI1621 & 2.218 & $0.152 * *$ & 0.358 & $4.267 *$ & $15.658^{* *}$ & -0.785 \\
\hline QLD65 X HD3237 & $4.531 * *$ & $0.131 * *$ & $1.820 * *$ & -1.706 & -4.208 & -0.57 \\
\hline QLD65 X PBW725 & -0.412 & -0.073 & 0.445 & 2.294 & 1.755 & 1.527 \\
\hline QLD65 X HI1621 & $-4.118 *$ & -0.057 & $-2.265 * *$ & -0.588 & 2.453 & -0.957 \\
\hline NW6036 X HD3237 & $-6.476^{* *}$ & $-0.250 * *$ & $4.114 * *$ & $5.707 * *$ & $16.993 * *$ & -0.307 \\
\hline NW6036 X PBW725 & -0.558 & 0.056 & $-3.316^{* *}$ & 1.354 & -0.208 & 1.275 \\
\hline NW6036 X HI1621 & $7.034^{* *}$ & $0.195 * *$ & -0.798 & $-7.061 * *$ & $-16.785 * *$ & -0.969 \\
\hline K1402 X HD3237 & 0.586 & $0.392 * *$ & $-1.234 *$ & $4.175 *$ & 2.611 & $2.140 *$ \\
\hline K1402 X PBW725 & $-6.567 * *$ & -0.077 & $2.000^{* *}$ & -0.744 & 2.45 & -1.18 \\
\hline K1402 X HI1621 & $5.981 * *$ & $-0.315 * *$ & -0.766 & -3.431 & -5.062 & -0.96 \\
\hline VORB/4/D67.2/PARANA 66.270 X HD3237 & $5.425^{* *}$ & -0.089 & $-2.739 * *$ & 0.425 & 1.45 & 0.781 \\
\hline VORB/4/D67.2/PARANA 66.270 X PBW725 & -1.603 & $0.248 * *$ & $-2.725 * *$ & 2.841 & 1.663 & 1.588 \\
\hline VORB/4/D67.2/PARANA 66.270 X HI1621 & $-3.822 *$ & $-0.159 * *$ & $5.464 * *$ & -3.266 & -3.113 & $-2.369 * *$ \\
\hline HPBW01 X HD3237 & 0.998 & -0.019 & $-2.811 * *$ & $-4.270^{*}$ & -9.491 & -1.295 \\
\hline HPBW01 X PBW725 & $4.262 * *$ & 0.015 & $3.627^{* *}$ & -1.549 & -3.845 & -0.24 \\
\hline HPBW01 X HI1621 & $-5.260 * *$ & 0.004 & -0.816 & $5.819 * *$ & $13.336^{* *}$ & 1.534 \\
\hline NAC/TH.AC//3*PVN/3/MIRLO/BUC/4/2*PASTOR/5/... X HD3237 & -0.979 & 0.04 & -0.202 & $9.237 * *$ & $18.054 * *$ & $2.663 * *$ \\
\hline NAC/TH.AC//3*PVN/3/MIRLO/BUC/4/2*PASTOR/5/... X PBW725 & $3.260^{*}$ & $-0.099 *$ & $-1.504 *$ & -2.192 & -5.934 & -0.165 \\
\hline NAC/TH.AC//3*PVN/3/MIRLO/BUC/4/2*PASTOR/5/... X HI1621 & -2.281 & 0.06 & $1.706^{* *}$ & $-7.045 * *$ & $-12.120 *$ & $-2.499 * *$ \\
\hline CD95\%SCA & 3.142 & 0.095 & 1.232 & 3.585 & 9.557 & 1.616 \\
\hline SE of SCA & 1.5727 & 0.6168 & 1.7945 & 0.0473 & 0.8087 & 1.3299 \\
\hline $\mathrm{SE}\left(\mathrm{s}_{\mathrm{IJ}}-\mathbf{s}_{\mathrm{kl}}\right)$ & 2.2242 & 0.8723 & 2.5378 & 0.0669 & 1.1437 & 1.8808 \\
\hline $\operatorname{SE}\left(s_{I J}-s_{i k}\right)$ & 4.4484 & 1.7446 & 5.0756 & 0.1338 & 2.2874 & 3.7615 \\
\hline
\end{tabular}

296

Environment Conservation Journal 
lines QLD 75 (0.325) followed by K 1402 (0.151) exhibited significant positive GCA for the trait.

In terms of SCA effects, values for the trait ranged between -0.315 to 0.392 . Seventeen hybrids were observed to possess significant SCA effects. The cross combinations K 1402 x HD 3237 (0.392) and VORB/4/D67.2/PARANA66.270 x PBW 725 (0.248) expressed high significant positive SCA effects for the trait.

\section{0 grain weight $(\mathrm{g})$}

GCA effects for 1000 grain weight among the parental lines were varying from -2.029 to 2.865 . Significant positive GCA effects were observed in the

line

NAC/TH.AC//3*PVN/3/MIRLO/BUC/4/2*PASTO

$\mathrm{R} / 5 / \ldots$ (2.865) followed by QLD 75 (1.839).

Whereas, SCA effects for the trait varied from 3.316 to 5.464 among the crosses. Twenty-two exhibited significant SCA effects for the character. The highest significant positive SCA effects were observed in the hybrid VORB/4/D67.2/PARANA66.270 x HI 1621 (5.464) followed by NW 6036 x HD 3237 (4.114).

\section{Biological yield per plant (g)}

GCA effects for the trait biological yield per plant ranged from -10.177 to 11.14 . Seven parental lines expressed significant GCA effects for the trait. Parental lines QLD 65 (11.164) followed by QLD 75 (7.325) and QBP 12-11 (6.075) showed significant positive GCA for the trait.

For biological yield per plant SCA effects ranged from -16.785 to 18.054 , Nine out of thirty-three F1s expressed significant SCA effects. The cross combination NAC/TH.AC// $3 * \mathrm{PVN} / 3 / \mathrm{MIRLO} / \mathrm{BUC} / 4 / 2 * \mathrm{PASTOR} / 5 / \ldots \mathrm{x}$ HD 3237 (18.054) followed by NW 6036 X HD 3237 (16.993) exhibited high significant positive SCA effects for the trait.

\section{Grain yield per plant (g)}

GCA effects for grain yield per plant ranged from 5.389 to 3.164. Five parental lines out of fourteen were observed with high significant positive GCA effects. The line QLD 75 (3.164), followed by SOKOLL (2.888), QLD 65 (2.819), QBP 12-11 (2.73) and HI 1621 (1.999) exhibited high significant positive GCA effects for the trait.

In terms of SCA effects the values for the trait observed between -7.250 to 9.237. Fourteen out of thirty-three crosses were observed to possess significant SCA effects for the trait. The cross combination

NAC/TH.AC//3*PVN/3/MIRLO/BUC/4/2*PASTO R/5/. . x HD 3237 (9.237), followed by HPBW 01 x HI 1621 (5.819), SOKOLL x HI 1621 (4.763), WH 1182 x PBW 725 (4.763) and QLD 75 x PBW 725 (3.781) exhibited significant positive SCA effects.

\section{Harvest Index}

Parental lines varied in a range from -1.35 to 1.392

for the trait harvest index. Five parental lines showed significant GCA effects for the character. Lines SOKOLL (1.392) followed by QLD 75 (1.352) were observed to possess significant positive GCA for the trait.

SCA effects among the crosses ranged from -3.358 to 2.806 for harvest index. Twelve F1s showed significant SCA effects. The highest significant positive SCA effects were demonstrated by the cross QLD $75 \times$ HI 1621 (2.806) followed by NAC/TH.AC//3*PVN/3/

MIRLO/BUC/4/2*PASTOR/5/.. x X HD 3237 (2.663). The results presented here are in accordance with earlier observations of Singh et al. (2013), Barot et al. (2014), Arya et al. (2018) and Patel et al. (2020).

The results highlighted from the present research investigation are in close confirmation with the earlier observations of Singh et al. (2013), Din et al. (2020) and Patel et al. (2020) for maturity traits; Kalhoro et al. (2015), Arya et al. (2018) and Patel et al. (2020) for tillers per plant, spikelets per spike, biological yield, spike length and grain yield per plant and Lohithaswa et al. (2014), Kalhoro et al. (2015) and Jatav et al. (2017) for harvest index (\%), grains per spike and grain weight per spike.

\section{Conclusion}

The present research investigation revealed that there is ample opportunity to exploit the genotypes in terms of combining ability. The parental lines VORB/4/D67.2/PARANA66.270,

NAC/TH.AC//3*PVN/3/MIRLO/BUC/4/2*PASTO R/5/..., PRL/2*PASTOR*2//FH6-1-7/3/ KINGBIRD\#1//... and QBP 12-1 were observed to be the superior parents for most of the traits including grain yield based on overall performance along with GCA and are recommended for further utilization in the future breeding programmes. In 
terms of cross combinations

VORB/4/D67.2/PARANA66.270 x PBW 725 was observed to be the superior hybrid for most of the traits, while, the cross combination NAC/TH.AC//3*PVN/3/MIRLO/BUC/4/

\section{References}

Arya, V.K., Singh, J., Kumar, L., Kumar, R., Kumar, P., \& Chand, P. (2018). Genetic variability and diversity analysis for yield and its components in wheat (Triticum aestivum L.). Indian Journal of Agricultural Research, 51(2): 128134.

Barot, H. G., Patel, M. S., Sheikh, W. A., Patel, L. P., \& Allam, C. R. (2014). Heterosis and combining ability analysis for yield and its component traits in wheat [Triticum aestivum (L.)]. Electronic Journal of Plant Breeding, 5(3), 350-359.

Bonjean, A.P., Angus, W.J., Van Ginkel, M., Pagesse, P., \& Moore, R.F. (2001). The World Wheat Book: A History of Wheat Breeding. Vol. 3. Lavoisier, Paris pp. 851-879.

Din, K., Khan, N. U., Gul, S., Khan, S. U., Tahir, I., Bibi, Z., .. \& Mumtaz, O. (2020). Combining ability effects and inheritance of maturity and yield associated traits in F2 populations of wheat. The Journal of Animal \& Plant Sciences, 30(4), 988.

Ismail, S. K. (2015). Heterosis and combining ability analysis for yield and its components in bread wheat (Triticum aestivum L.). International Journal of Current Microbiology and Applied Sciences, 4(8), 1-9.

Jatav, S. K., Baraiya, B. R., \& Kandalkar, V. S. (2017) Combining ability for grain yield and its components different environments in wheat. Int. J. Curr. Microbiol. App. Sci, 6(8), 2827-2834.

Kalhoro, F. A., Rajpar, A. A., Kalhoro, S. A., Mahar, A., Ali, A., Otho, S. A., ... \& Baloch, Z. A. (2015). Heterosis and combing ability in F1 population of hexaploid wheat (Triticum Aestivum L.). American Journal of Plant Sciences, 6(07), 1011.

Kempthorne, O. (1957). An introduction to genetic statistics.

Lohithaswa, H. C., Desai, S. A., Hanchinal, R. R., Patil, B. N., Math, K. K., Kalappanavar, I. K., ... \& Chandrashekhara, C. P. (2014). Combining ability in tetraploid wheat for yield, yield attributing traits, quality and rust resistance over environments. Karnataka Journal of Agricultural Sciences, 26(2).
2*PASTOR/5/.... x HD 3237 was showing better performance for grain yield. Therefore, these cross combinations may be further exploited for the isolation of suitable transgressive segregants in a breeding programme.

Patel, P. U., Patel, B. C., Sidapara, M. P., \& Sharma, D. D. (2020). Combining Ability and Gene Action Studies for Yield and its Component Traits in Bread Wheat (Triticum aestivum L.). Int. J. Curr. Microbiol. App. Sci, 9(5), 24632469.

Prasad, B. (2014). Heterotic vigour studies in forage sorghum hybrid by multiple criteria. Journal of Hill Agriculture, 5(2), 182-185.

Saeed, M., Khalil, I. H., Anjum, S. A., \& Tanveer, M. (2016). Combining ability and heritability for yield traits in wheat (Triticum aestivum L.). Pakistan Journal of Agricultural Sciences, 53(3).

Sarwar, I. (2016). Detection of gene action and combining ability effects in bread wheat (Triticum aestivum L.) using line $\mathrm{X}$ tester scheme.

Singh, B. D. 2015. Plant Breeding: Principles and methods. Kalyani Publishers, New Delhi. pp. 203-205.

Singh, G. P., Sendhil, R., \& Jasrotia, P. (2019). AICRP on wheat and barley salient achievements and future directions. Ind J Fertilizers, 15(4), 80-90.

Singh, M. A. M. T. A., Devi, E. L., Aglawe, S., Kousar, N., \& Behera, C. (2013). Estimation of heterosis in different crosses of bread wheat (Triticum aestivum L.). The Bioscan, 8(4), 1393-1401.

Tabassum, Kumar, A., \& Prasad, B. (2017). Study of Combining Ability and Nature of Gene Action for Yield and Its Contributing Traits in Bread Wheat (Triticum aestivum L. em. Thell). International Journal of Current Microbiology and Applied Sciences, 6(10): 3562-3573.

Thakre, J. M., Vitkare, D. G., Godbole, A. K., \& Atale, S. B. (1996). Line X Tester analysis in wheat for yield and it's traits. Annals of Plant Physiology, 10, 99-102.

Younas, A., Sadaqat, H. A., Kashif, M., Ahmed, N., \& Farooq, M. (2020). Combining ability and heterosis for grain iron biofortification in bread wheat. Journal of the Science of Food and Agriculture, 100(4), 1570-1576. 